\title{
High-sensitive Biomarkers of Blood Antiradical Activity in Mice Exposed to $\gamma$-irradiation
}

\author{
S. Kalmakhelidze ${ }^{1,3}$, T. Sanikidze ${ }^{* 1,4}$, D. Topuria ${ }^{2}$, I. Chkhikvishvili ${ }^{1,6}$, E. Shekiladze ${ }^{1,5}$, N. Ivanishvilli ${ }^{4}$, \\ M. Gogebashvili ${ }^{4}$, E. Lomadze ${ }^{1,4,5}$, G. Ormotsadze ${ }^{1,4}$ \\ ${ }^{1}$ Department of Medical Physics and Biophysics, Tbilisi State Medical University, Tbilisi/ 0177, Georgia \\ ${ }^{2}$ Department of Normal Anatomy Tbilisi State Medical University, Tbilisi/0177, Georgia \\ ${ }^{3}$ Department of Neurotoxicology, Beritashvili Center of Experimental Biomedicine, Tbilisi/0160, Georgia \\ ${ }^{4}$ Department of Radiobiology, Beritashvili Center of Experimental Biomedicine, Tbilisi/0160, Georgia \\ ${ }^{5}$ Department of Biophysics, Javakhishvili Tbilisi State University, Tbilisi/0167, Georgia \\ ${ }^{6}$ V. Bakhutashvili Institute of Medical Biotechnology of Tbilisi State Medical University, Tbilisi/0159, Georgia \\ *Corresponding author: Tamar Sanikidze; tsanikidze@tsmu.edu
}

Received 15 February 2021;

Accepted 08 March 2021;

Published 16 March 2021

\begin{abstract}
Diagnostic markers are important indicators for the assessment of the possible consequences of radiation exposure, which could lead to acute and chronic radiation toxicity. The goal of the study is to determine high-sensitive markers of blood redox status in relation to the dose of radiation.

The mice were exposed to $\gamma$-irradiation (Cs137) at a total dose of 3, 5, and 7Gy. After 1, 2, 7, and 14 days of the irradiation, the blood samples were drawn under ether anesthesia from the inferior vena cava and the activity of antioxidant system (enzymatic -superoxide dismutase (SOD), catalase) and non-enzymatic total antioxidant activity (TAA) of blood serum was determined.

The results of our investigation support the fact that the non-enzymatic antioxidant TAA plays an important role in the prevention of radiation damage during ionizing radiation exposure, which makes it possible to consider TAA as a promising candidate as the biomarker of radiation dose.
\end{abstract}

Keywords: $\gamma$-radiation, antiradical system, biodosimetry markers

\section{Introduction}

Major advances in medical and supportive care have contributed to a growing population of cancer survivors. Most patients diagnosed with cancer today are expected to become long-term survivors. Unfortunately, improved prognosis has been accompanied by longterm health problems due to cancer treatment. Approximately $75 \%$ of survivors experience one or more adverse events, including severe cardiac complications ${ }^{[1,2]}$. This circumstance was reflected in ICRP Publication $118^{[3]}$. Based on this recommendation, new, highly effective, and inexpensive biomarkers of the individual risk for tissue reactions and strategies to prevent/mitigate tissue effects after exposure are a modern priority in radiation biomedicine ${ }^{[4]}$. Modern approaches to the search for absorbed dose (dose marker), radio-induced shifting effect, and marker of susceptibility in the conditions of the fractional partial body irradiation are concentrated on complex - genetic, cytogenetic, metabolic characteristics ${ }^{[5,6]}$. In parallel with the search of gene candidates of biodosimetry markers of radiation exposure ${ }^{[7]}$, the study of traditional, redox status imbalance biomarkers is also underway ${ }^{[8]}$.

$\gamma$-radiation hazardous impacts on living tissue such as DNA damage, genomic instability, apoptosis, and inflammation is mediated by the generation of reactive oxygen species (ROS), (hydroxyl radical, superoxide anion, and hydroperoxyl radicals) generated by the radiolysis of water ${ }^{[8,9]}$. After irradiation, the chronic inflammatory response includes the activation of kinases, transcription factors, and the production of inflammatory cytokines ${ }^{[10]}$, as well as the accumulation of free radicals and ROS. Excessive formation of ROS shifts the normal redox equilibrium of the cell towards the oxidized state and results in an imbalance between pro-oxidative and antioxidative reactions, depletion of antioxidant capacity leading to negative consequences in metabolism ${ }^{[11]}$.

Specialized enzymes (catalase (Cat), superoxide dismutase (SOD), glutathione system enzymes, etc.), as well as, numerous non-enzymatic low molecular weight antioxidants (ascorbic acid, 
bilirubin, estrogens, biogenic amines (dopamine, histamine, serotonin, melatonin, etc.) prevent excessive formation of free radicals in eukaryotic cells. In the existing scientific literature is available data on the activity of the antioxidant enzyme system in the early stages of irradiation - it was found that ionizing irradiation led to increased antioxidant enzymes activity within a few minutes ${ }^{[12]}$ and decreased to the control or lower level after 24-48 hours after irradiation ${ }^{[13]}$. As for the non-enzymatic antioxidant system and its role in the mechanisms of radiation damage in the first hours after irradiation few research data is available ${ }^{[15]}$.

In our research, we studied the nature of the causal relationship between the final biological effect of ionizing radiation exposure and the dynamics of the antioxidant system activity in the post-radiation period. The goal of this study was to determine highsensitive markers of the blood redox status in relation to the radiation dose, which will facilitate the assessment of the level of radiation toxicity. The survival time of animals in the posttraditional period was used as the final criterion for the radiobiological effect.

\section{Materials and Methods}

The experimental protocol was in accordance with the guidelines for care and use of laboratory animals as adopted by the Ethics Committee of the Tbilisi State Medical University (TSMU).

\section{Animal care and maintenance}

Six - to seven-week-old male mice (Mus musculus), were obtained from Vivarium of Tbilisi State Medical University. They were housed in animal cages, with room temperature maintained at $200220 \mathrm{C}$, relative humidity of 50-70\% and an airflow rate of 15 exchange/h. Also, a time-controlled system provided 08:00-20:00 h light and 20:00-08:00 h dark cycles. All mice were given standard rodent chow diet and water from sanitized bottle fitted with stopper and sipper tubes.

\section{Experimental design}

After acclimatization for a week to laboratory conditions, the mice were divided into four different groups (total of 200 mice with 50 rats in each group). Mice whole-body irradiation with 137Cs (Cesium-137) was performed at a dose rate of $1,1 \mathrm{~Gy} / \mathrm{sec}$ for the total doses of 3, 5 and 7Gy (Group 1, 2, and 3) with a "Gamma cell 40 Exactor" (MDS Nordion, Ottawa, Canada). The sham-irradiated animals (Group 4) were placed in the irradiator for the same amount of time, but they were not exposed to the source. Subsequently, the sham and irradiated mice were transferred to a regular holding room. Following exposure to gamma irradiation, six mice from each group were sacrificed at 1 ( $24 \mathrm{~h}), 2$ (48 h), 7 and 14 days. Blood samples were collected from the inferior vena cava under ether anesthesia. The survival time of animals in the post-radition period was used as the final criteria for the radiobiological effect.

\section{Biochemical parameters}

The blood was centrifuged at 2,000g for $10 \mathrm{~min}$; Blood serum was separated in nonheparinized tube for serum collection (from 2 mice in each sample) and the activity of antioxidant enzymes, Cat and SOD was determined with standard methods by spectrophotometry with ELISA Microplate Reader (Bio Tek Instruments, Inc, USA). The activity of SOD was determined by absorbance at $450 \mathrm{~nm}$ and expressed as percent of inhibition rate (inhibition rate \%); activity of Cat was measured at $570 \mathrm{~nm}$ and expressed as $\mathrm{mU} / \mathrm{mL}$
Total activity of low molecular weight non-enzymatic antioxidants (TAA) was determined in deproteinized blood plasma by using the 2.2-diphenyl-1-picrylhidrazyl (DPPH)-scavenging assay, which was adapted from study conducted by Chrzczanowicz et al. ${ }^{[14]}$. Briefly, plasma samples $(1 \mathrm{~mL})$ were deproteinized by adding $3 \mathrm{~mL}$ of acetonitrile and centrifuging then for $10 \mathrm{~min}\left(4^{\circ} \mathrm{C}\right.$, $9500 \times \mathrm{g}$ ). A supernatant was immediately collected and $1 \mathrm{~mL}$ was transferred to a tube. Subsequently, $3 \mathrm{~mL}$ of DPPH was added, and the resultant absorbance was read at $515 \mathrm{~nm}$. A calibration curve was built with the use of gallic acid, wherein the absorbance values were interpolated and the results were expressed as equivalents of gallic acid.

\section{Statistical analysis}

Each data point represents mean standard error on the mean (SEM) of at least six animals per group. $\mathrm{P}<0.05$ was considered to represent a statistically significant difference. The analysis and visualization of data was conducted by using "SPSS-12"for Windows.

\section{Results}

Figure 1 shows the survival curves of mice irradiated with $7 \mathrm{~Gy}$ (LD 100/30), 5Gy (LD50/30), and 3Gy (LD50) doses. As shown in the Figure 1, animals irradiated with dose 7Gy die within the first twenty days after irradiation, $50 \%$ of animals irradiated with dose 5Gy die 30-35 days after irradiation. In the case of animal irradiation with $3 \mathrm{~Gy}$ dose, the death of animals started on the 40th50 th day, and $50 \%$ of the animals died on the 180th day after irradiation; after that, the intensity of animal deaths was not reliably different from the control group animal death.

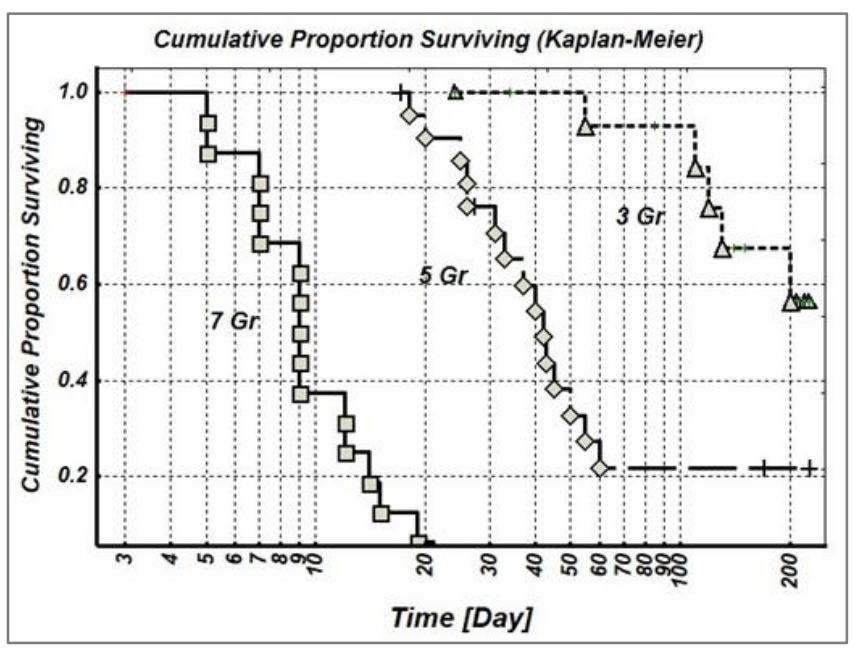

Figure 1: Survival function (Kaplan-Meier plot with censored data) of mice irradiated with 3, 5 and $7 \mathrm{Gr}$ (Horizontal axis Postradiation period interval [Log (Day)], Vertical axis - survival probability).

The dynamics of the activity of antioxidant enzymes and TAA in irradiated mice are shown in Figs. 2a, b, c. As can be seen in Fig. 2a SOD activity of the irradiated mice (3Gy) (group 1) decreased significantly in comparison with the control group 24 and $48 \mathrm{~h}$ after irradiation $(\mathrm{p}<0.05)$. On the 7 th day of observation, the activity of SOD increased, and on the 14th day, it reached the initial level. SOD activity of mice irradiated with 5-7Gy (groups 2, 3) 24 and $48 \mathrm{~h}$ after irradiation was significantly lower than in the group irradiated with $3 \mathrm{~Gy}$ irradiated group $(\mathrm{p}<0.05)$. On the 7 th day, the activity of SOD continued to decrease in both groups (groups 2. 3), but on the 14th day, it slightly increased in animals 
of group 2 and reached $29 \%$ of the initial level in mice of $3 \mathrm{rd}$ group.

As shown in Fig. 2b, the activity of Cat in irradiated mice (3Gy) (group 1) at 24 and 48 hours after irradiation compared to the control group $(\mathrm{p}<0.05)$. On the 7 th day of observation, the activity of Cat increased, and on the 14th day, it reached the initial level. The activity of Cat activity in mice irradiated with 5-7Gy (groups 2,3) was significantly lower as than in the group irradiated with $3 \mathrm{~Gy}(\mathrm{p}<0.05)$. On the 7 th day the activity of Cat continued to decrease in both groups (groups 2,3), but on the 14th day did not change in animals of group 2 and continued to decrease in mice of group 3.

As shown in Fig. 2C, TAA of irradiated mice (3, 5, 7Gy) decreased significantly after $24 \mathrm{~h}(\mathrm{p}<0.05)$ and begin to increase on the second day after irradiation when compared to the control group. TAA of mice irradiated with 5-7Gy at 24 and $48 \mathrm{~h}$ after irradiation was significantly lower as in the group irradiated with $3 \mathrm{~Gy}(\mathrm{p}<0.05)$. In mice irradiated with 3-5Gy, TAA began to increase after $48 \mathrm{~h}$, continued to grow, and reached the control level on the 14th day of observation. In animals irradiated with 7Gy TAA significantly decreased the 14th day.

The activity of SOD, Cat, and TAA of the sham irradiated animals (group 4) did not differ from the control group (data not shown).
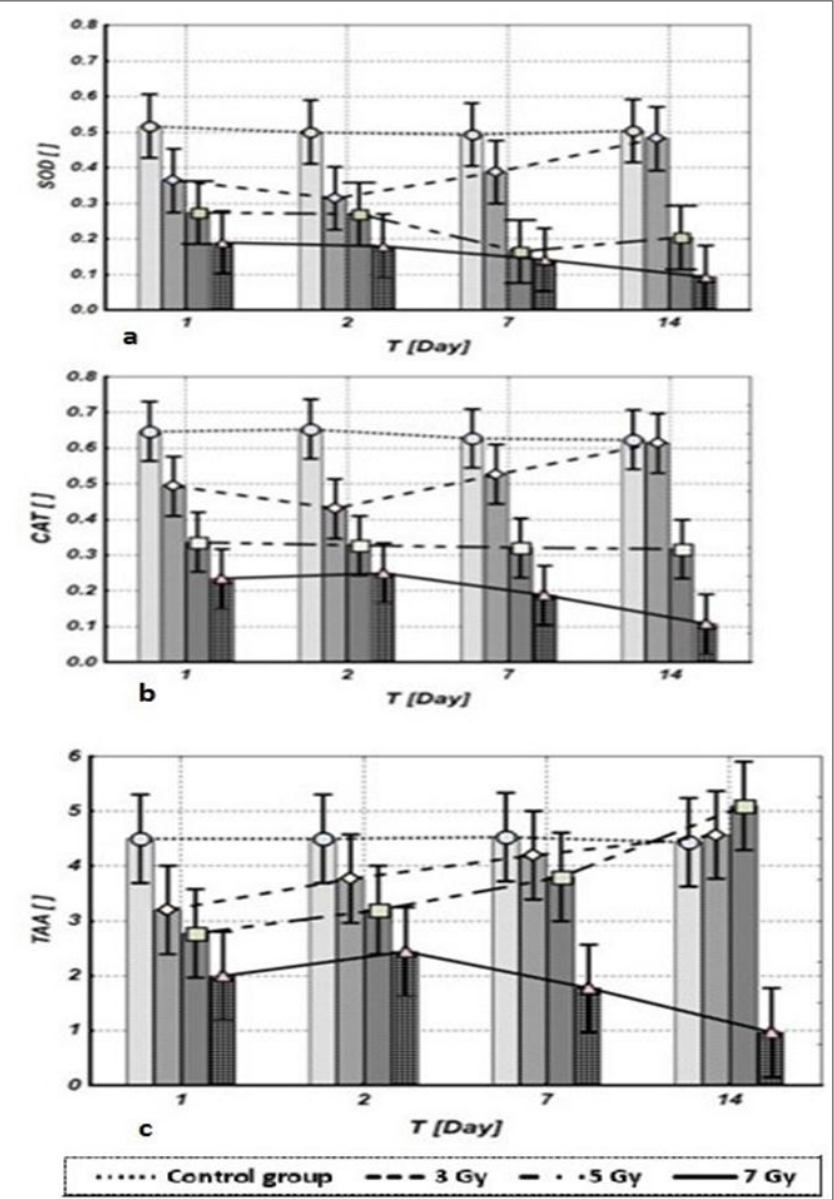

Figure 2: Dynamics (1, 2, 7 and 14 days) of the activity of SOD (a), Cat (b) and TAA (c) in the blood of gamma irradiated mice (at doses of 3, 5 and 7 Gy)

\section{Discussion}

The aim of this study was to determine the causal relationship between the final biological effect of ionizing radiation exposure and the dynamics of the activity of antioxidant system of mice in the post-radiation period.

In response to the direct effect of ionizing radiation on cellular targets, in the result of radiolysis of water, as well as due to the compensatory response of the body to primary damage (increased activity of mitochondrial and microsomal electron transport systems) in animal tissues produced a huge amount of ROS. Against the increased formation of ROS in the body the enzymatic and the non-enzymatic antioxidant system ensure the neutralization of free radicals. The ability of cells in a living organism to prevent specific and non-specific oxidative damage is a key survival mechanism.

Activation of lipid peroxidation, accumulation of peroxides, inactivation of antioxidant enzymes, and inhibition of the biosynthesis of macromolecules during the period of primary radiation injury restricts the replenishment of the pool of the enzymatic antioxidant system of the body. Animal studies revealed a dose-dependent increase in the content of oxidation products (lipoperoxides, malondialdehyde) after whole-body irradiation with doses 2, 4, and 6Gy ${ }^{[12]}$. A few minutes (5-15 min) after irradiation, compensatory activation of antioxidant enzymes (SOD, Cat) was observed in the absence of their increased transcription ${ }^{[15,16]}$, which further contributes to the depletion of the antioxidant enzymatic system. Indeed, the results of our study show that in 24$48 \mathrm{~h}$ after mice whole-body gamma-irradiation with doses 3,5 , and $7 \mathrm{~Gy}$, the activity of the enzymatic antioxidant system (SOD, Cat) dose-dependently decreased which may be related to their utilization in the processes of detoxication of excess ROS (an excess amount of superoxide radicals can reduce the activity of SOD, and hydrogen peroxide formed as a result of detoxification of superoxide radicals inhibits catalase). The total activity of the nonenzymatic antioxidant system (TAA) also dose-dependently decreased during the first 24 hours after irradiation and then began to increase.

Thus, within 1-2 days after irradiation, the range of changes in the activity of SOD, Cat, and TAA depends on the dose of irradiation, the dynamics of these dose-dependent changes are similar. Accordingly, in the early post-radiation period, the severity of post-radiation disorders in the body depends on the radiation dose and subsequently determines the intensity of systemic adaptive response, in particular, restoration of energy supply, detoxification of ROS, and biomolecule synthesis.

In the subsequent post-radiation period (3-14 days) in mice irradiated with a low dose (3Gy), a compensatory increase in the activity of SOD and Cat in the blood was observed, which should be associated with intensification of synthetic processes and an increase in the pool of antioxidant enzymes in the body. However, in the case of high doses (5Gy, 7Gy), the compensatory replenishment of the pool of antioxidant enzymes against the background of the intensive formation of ROS was insufficient, which was revealed by a dose-dependent decrease in the SOD and Cat activity (especially at irradiation with 7Gy (Fig.2c)).

At the same time, resistance to radiation damage largely depends on the individual variability of the antioxidant system: Hartmeyer et al (1997) showed that the activity of SOD and Cat increased within $15 \mathrm{~min}$ after exposure to radiation (2-6Gy) in radiation-resistant but not in radiation-sensitive mice ${ }^{[12]}$. The results of our previous studies also indicate a high variability of antioxidant enzyme activity in the post-radiation period ${ }^{[13]}$.

The TAA activity statistically significantly increases within 2-14 days after irradiation with doses of 3-5Gy and reaches the control values on the 14th day of observation, while in the case of irradiation with a dose of 7Gy, TAA sharply decreases on the 3- 
$14^{\text {th }}$ day of observation (Fig.2a,b,c). The dynamics of dosedependent alterations in the indicators of the TAA correlates with the data of the survival curve of the irradiated animals (Fig.1,2) which confirms the assumption, that the non-enzymatic antioxidant system represents a key mechanism of antiradical defense in radiated animals and allows to considerate TAA as a promising biomarker of the irradiation dose.

\section{Conclusion}

The prospect of the use of new characteristics of the general antiradical status of the organism as a potential biomarker of the effects of radiation exposure was examined. The results support that the non-enzymatic antiradical system plays an important role in the prevention of radiation damage during ionizing radiation exposure and make it possible to consider TAA as a promising biomarker in the determination of dose and the effect of radiation exposure which can be used in radiotherapy procedures to detect and prevent possible complications.

\section{References}

[1] I.B. Balazs and T. Phillip, "Breast Cancer Survivorship: A Comprehensive Review of Long-Term Medical Issues and Lifestyle Recommendations", The Permanente Journal, vol. 19, no.2, pp.48-79, 2015.

[2] C.S. Darby, M. Ewertz, P. McGale et al, "Risk of Ischemic Heart Disease in Women after Radiotherapy for Breast Cancer", N Engl J Med, no. 368, pp. 987-98, 2013.

[3] ICRP publication 118: ICRP statement on tissue reactions and early and late effects of radiation in normal tissues and organs--threshold doses for tissue reactions in a radiation protection context. Ann ICRP, vol. 41, no. 12, pp. 1-322, 2012.

[4] ICRP 2013: 2nd International Symposium on the System of Radiological Protection. Abu Dhabi. October 2013.

[5] M. Sproull, T. Kramp, A. Tandle et al, "Analysis of Radiation Responsive Proteins to Predict Radiation Exposure in Total-Body Irradiation and Partial-Body Irradiation Models", Radiation Research, vol.187, no.2, pp. 251-258, 2017.

[6] C. Herate and L. Sabatier, "Retrospective biodosimetry techniques: Focus on cytogenetics assays for individuals exposed to ionizing radiation in: Klein CB, Parsons BL", Mutation Research. Vol. 783, pp.108-287, 2020.

[7] J. Lacombe, C. Sima, S.A.Amundson et al, "Candidate gene biodosimetry markers of exposure to external ionizing radiation in human blood: A systematic review", PLOS ONE, vol. 13, no. 6, Article ID e0198851, 2018.

[8] Bryll, W. Krzy'sciak, A. Jurczak et al, "Changes in the Selected Antioxidant Defense Parameters in the Blood of Patients after High Resolution Computed Tomography", Int. J. Environ. Res. Public Health, vol 16, no 9, Article ID 1476, 2019.

[9] W. Zhao, D.I. Diz and M.E. Robbins, "Oxidative damage pathways in relation to normal tissue injury", Br. J. Radiol, vol. 80, no. 1, pp. S23-S31, 2007

[10] M. Hazbavi, M. Zarei, R. Nazaralivand et al, "Protection from ionizing radiation-induced genotoxicity and apoptosis in rat bone marrow cells by HESA-A: a new herbal-marine compound", Journal of Bioenergetics and Biomembranes, vol. 51, pp. 371-79, 2019

[11] R. Hardmeier, H. Hoeger, S. Fang-Kircher et al, "Transcription and activity of antioxidant enzymes", PNAS, vol. 94, no. 14, pp. 7572-76, 1997.

[12] D. Ujjal, M. Krishnendu, S. Mahuya et al, "Role of Ferulic Acid in the Amelioration of Ionizing Radiation Induced Inflammation: A Murine Model", PLOS ONE, vol. 9, no. 1, Article ID e97599, 2014.

[13] C.K. Robinson, K. Webb, A. Kaur et al, "Major Role for Non-Enzymatic Antioxidant 1 Processes in the Radioresistance of Halobacterium salinarum", J Bacteriol, vol. 93, pp. 1653-62, 2011.

[14] J. Chrzczanowicz, A. Gawron, A. Zwolinska et al , "Simple method for determining human serum 2,2diphenyl-1-picryl-hydrazyl (DPPH) radical scavenging activity - possible application in clinical studies on dietary antioxidants", Clinical Chemistry and Laboratory Medicine, vol. 46, pp.342-349, 2008

[15] Gvilava, G. Ormotsadze, I. Chkhikvishvili et al, "Radioprotective activity of polimetoxy-lated flavonoids of citrus extract", Georgian Med News, vol. 12, no. 285, pp. 119-124, 2012.

[16] Gvilava, I. Chkhikvishvili, T. Sanikidze et al, "Study of total antioocidant status of the organism as a possible biomarker of dose and effect of radiation exposure", Georgian Medical News, vol. 5, no. 278, pp.177-183, 2018. 